Military Technical College, Kobry El-Kobbah, Cairo, Egypt

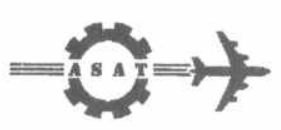

$9^{\text {th }}$ International Conference

On Aerospace Sciences \& Aviation Technology

\title{
AIRCRAFT RECOGNITION SYSTEM USING A SIMPLE INVARIANT COMPLEX-NEURAL NETWORK
}

\section{Abstract:}

Bahgat S.F.*

Aircraft image recognition is an important sub-problem of photo-interpretation, which continues to be a major application of domain of image understanding techniques. This paper describes a new approach to aircraft image recognition system. This approach combines complex moments with complex neural-network. For aircraft recognition using complex moments are a recent one of the most useful features that can be extracted from an image because they can be invariant to translation, rotation, and scaling of the object. A neural network systems are adept at many pattern recognition tasks which require the ability to match large amount of input information simultaneously and then generate categorical or generalized output. Neural network systems posses these capabilities as well as the ability to learn and build unique structures specific to a particular problem, so they are especially useful in pattern recognition. This paper presents complex neural classifier, which uses complex moment function as inputs. This approach differs significantly from many recent aircraft recognition system, which emphasize the geometric features of objects in the scene, experimental results are given to illustrate the efficacy of this approach.

\section{Introduction}

An important aspect of human visual system is the ability to recognize an object despite changes in the object's translation, rotation, and scale. A machine vision system is the implementation of human visual system. The methods adopted for this purpose can be done, which exploit tactile information to build an analytical model of the explored object. Structural methods describe an object by means of grammar of constituent primitive parts and associated relationships. Neural networks (NN) have been shown to be effective as computational processors for various tasks including recognition of complex patterns (visual image recognition)[1]. Pattern recognition tasks require the ability to match large amounts of input information simultaneously and then generate categorical or generalized output. They also require a reasonable response to noisy or incomplete input. NN systems posses these capabilities as well as the ability to learn and build unique structures specific to a particular problem, so they are especially useful in pattern recognition. NN data is a parallel processing structural that has large numbers of processors and many inter connections between them. A typical NN neuron or computing element is basically a comparator that

\footnotetext{
* Associate Professor, Egyptian Armed Forces, Cairo, Egypt.
} 
produces an output when the cumulative effect of the input stimuli exceed a threshold value. NN has some properties or characteristics not exist in traditional expert systems such as learning, generalization, abstractions, and applicability. NN can be realized in hardware, image compression, and pattern recognition algorithms can be done in real time, since the networks are highly parallel. In other words, each neuron is independent of the other neuron at the same layer. NN is highly fault tolerant due to the massive interconnection between nodes in the network. Thus, a failure in one node or link will not significantly degrade the reference of the entire system [1]. Neural nets provide a greater degree of robustness and fault tolerance than conventional computers, because of the many processing nodes, each of which is responsible for a small portion of the task. Damage to a few nodes or links does not impair the overall performance significantly.

This paper presents complex neural network classifiers, which use complex moment functions as inputs. The neural networks are trained with feature vectors with complex moment invariant components to obtain the desired classification index at the output. The most frequently used networks for the above application is multi-layer perceptions and the classification schemes are implemented using multi-layer feed forward algorithms. The description of the proposed aircraft recognition system is defined in section 2. The feature vector of image and complex moment descriptor classifier algorithm are presented in section 3. Aircraft model and image capture is presented in section 4. The architecture of complex NN model and the complex NN training algorithm presented are presented in section 5 . Section 6 shows experimental results. Finally, Conclusions and suggestions for future work are presented in section 7 .

\section{Proposed Aircraft Recognition System:}

The structure of the proposed aircraft recognition system using simple invariant complex neural network is shown in Fig. (1). It consists of a sensor (an image sensor or camera), Image processing programs to enhance obtained images to be suitable for feature extraction, a feature extraction mechanism (algorithm), and a classification or description algorithm (complex neural network classifier). In addition, usually some data that have already been classified or described are assumed available in order to train the system (the so-called training set). Training set is very important for the proposed system because as the capacity of this set is increased the performance of the system for identification of the aircraft is increased. The sensor in the proposed system is either thermal or ordinary light camera, which is concerned with the process of translation from light stimuli (physical images) falling onto the photo sensors of a camera to a digital electrical value suitable for storing within the computer's memory. 


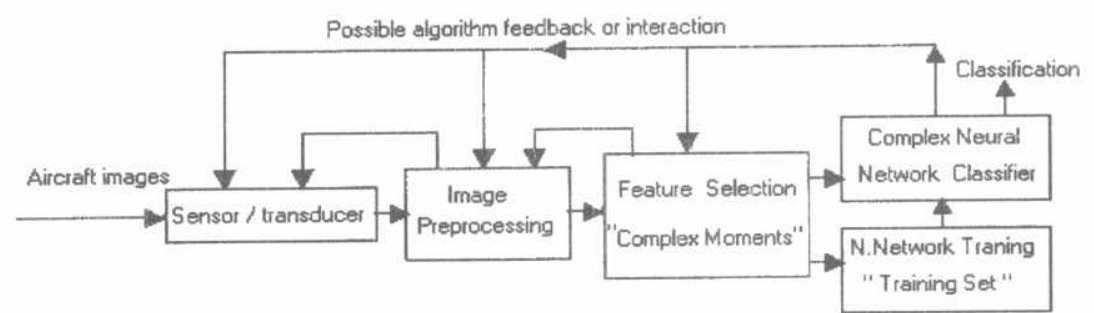

Fig. (1) Functional block diagram of the proposed recognition system

Typically a digital picture can be up to $512 \times 512$ pixel resolution with each pixel representing a binary, gray scale or color value. The choice of spatial and luminance resolution parameters critically affect the workload of the computer and therefore must be inimized while ensuring that no useful information is lost. After that preprocessing stage seek to modify and prepare the pixel values of the digitized image to produce a form that is more suitable for subsequent operations especially for feature extraction. Typically contrast enhancement and adjustment, filtering to remove noise and improve quality, and correction for sensor distortion may all be desirable during this stage. These operations only change the pixel values of the digitized image within the frame store, or subsequent display memory. The segmentation process is not primarily concerned with what the regions represent, but only with the process of partitioning the image. In the simplest case (binary images) there are only two regions: a foreground (object) region and a background region. Moments are one of the most useful features that can be extracted from an image (from the region) because they can be invariant to translation, change in size, intensity and rotation of the object. Higher order moments will be used in 2D-pattern and 3D-object recognition. Complex moments are a recent approach for driving moment invariant. They form an intermediate step between ordinary moments and moment invariant. [ 3 ].

In order to classify shapes (images) using complex moment functions, a feature vector of complex moments is presented at the input of the complex neural network, and the output is specified as the value of the output node being (1), and the remaining values (0). Our proposed recognition algorithm is given in terms of the third order complex moment for feature extraction, however, using any of the complex moment coefficients whose angular order is unity does not change any of the steps of the algorithm or the validity of the proof.

The steps to achieve this algorithm are as follows:

(1) Compute the complex moment invariant vector (CMV) to each aircraft,

(2) Present CMV at the input of complex neural network.

(3) Training NN with different images represented by the same feature vector.

(4) Test the neural network with another samples of images.

\section{Feature Extraction and Moment Invariant:}

One of the most difficult problems in the design of a shape recognition system relates to the selection of a set of appropriate numerical attributes or features to be extracted from the object of interest for purposes of classification. The success of any practical 
system depends critically upon this decision. Although there is little in the way of a general theory to guide in the selection of features for an arbitrary problem [6], it is possible to state some desirable attributes of features for recognition of shapes. Among these are the following:

1-The features should be informative. That is, the dimensionality of a feature vector should be as low as possible, consistent with acceptable recognition accuracy.

2-The feature should be invariant to translation, change in size, intensity and rotation of the object

Moments have been proposed as a solution to this problem in two ways [9]:

- Using moment invariant as features of the image that retain their values when the image is translated, scaled, or rotated.

- Using second order moments to normalize the image and then extracting suitable features to classify the normalized image.

Complex moments [3] have recently introduced to characterize the ability of moment invariants to discriminate between classes. We use the same formalism here to define a general class of normalization procedures using higher order moments. The establishment of normalization procedures based on higher order moments allows us to directly compare the invariance properties of moment invariants versus those of images that have been normalized through moments. In particular, we show that an image normalized in the manner we propose retains all the invariance properties of moment invariants in the presence of noise. The significance is that pattern recognition algorithms can be devised that operate on normalized images and in principle, have all the within-class insensitivity of moment invariants but not their shortcomings with regard to distinguishing between different classes.

\subsection{Geometric (Ordinary) Moments Definition:}

Given an image characterized by an interior intensity function $f(x, y)$ in a 2-D plane, the geometric (ordinary) moment of order $(p, q)$ is defined as [9] :

$$
M_{p \mathrm{q}}=\int_{-\infty}^{\infty} \int_{-\infty}^{\infty} x^{p} y^{q} \mathrm{f}(\mathrm{x}, \mathrm{y}) \mathrm{dx} \mathrm{dy}
$$

There are theorems which guarantee the existence and uniqueness of provided that $f(x, y)$ has a finite value. Central moments which are derived from the ordinary moments are translation invariant, $\mu_{\mathrm{p} \mathrm{q}}$

$$
\mu_{p \mathrm{q}}=\int_{-\infty}^{\infty} \int_{-\infty}^{\infty}(x-\bar{x})^{p}(y-\bar{y})^{q} \mathrm{f}(\mathrm{x}, \mathrm{y}) \mathrm{dx} \mathrm{dy}
$$

Where $\bar{x}, \bar{y}$ represents the centroid of the image and calculated as follows:

$$
\bar{x}=\frac{M_{10}}{M_{00}}, \bar{y}=\frac{M_{01}}{M_{00}}
$$

Analytical expressions useful in computing second and third order central moments directly from ordinary geometric moments are given below. 


$$
\begin{aligned}
& \mu_{02}=m_{02}-y_{0} m_{01} \\
& \mu_{20}=m_{20}-x_{0} m_{10} \\
& \mu_{11}=m_{11}-y_{0} m_{10} \\
& \mu_{30}=m_{30}-3 x_{0} m_{20}+2 x_{0}^{2} m_{10} \\
& \mu_{03}=m_{03}-3 y_{0} m_{02}+2 y_{0}^{2} m_{01} \\
& \mu_{21}=m_{21}-2 x_{0} m_{11}-y_{0} m_{20}+2 x_{0}^{2} m_{01} \\
& \mu_{12}=m_{12}-2 y_{0} m_{11}-x_{0} m_{02}+2 y_{0}^{2} m_{10}
\end{aligned}
$$

\subsection{Complex Moments Definition and Complex Moment Descriptor:}

The complex moments are a recent approach for deriving moment invariants. They form an intermediate step between ordinary moments and moment invariants $[9,10]$. Complex moment have a computational advantage and at a particular order they are less affected by noise than ordinary moments of the same order. The complex moments of order $(p, q)$ for two dimensional function $f(x, y)$ are given by:

$$
c_{p q}=\int_{-\infty-\infty}^{+\infty} \int_{-\infty}^{+\infty}(x+j y)^{p}(x-j y)^{q} f(x, y) d x d y
$$

If the contrast of the image, $f(x, y)$ is real non-negative, $c_{p p}$ is a real non-negative number, and $c_{p q}$ is the complex conjugate $c_{q p}$.

In polar coordinates the complex moment of $\operatorname{order}(p, q)$ is:

$$
c_{p q}=\iint r^{p+q} e^{j(p-q)} f(r, \theta) r d r d \theta
$$

Where: $f(r, \theta)=f(r \cos \theta, r \sin \theta)=f(x, y)$

it can be seen that when the image is rotated by an angle $\theta$ the new moments may be expressed by:

$$
c_{p q}^{\prime}=c_{p q} e^{i(p-q) \theta}
$$

The normalized central complex moments, $c_{p q}^{n c}$ can be calculated as follows:

$$
c_{p q}^{n c}=\left[\frac{1}{c_{00}}\left(\frac{c_{00}}{c_{11}}\right)^{\frac{p+q}{2}}\right] c_{p q}^{c}
$$

If $c_{p q}^{n c}=\left|c_{p q}^{n c}\right| e^{j \phi p q}$ then, define the complex moment descriptor $\mathrm{C} D_{p q}$,

$$
C D_{\mathrm{Pq}}=\left|C D_{p q}\right| e^{j \beta p q} \text {, as follows: }\left|C D_{p q}\right|=\left|c_{p q}^{n c}\right|, \beta_{p q}=\phi_{p q}-(p-q) \phi_{21}
$$

The complex moments descriptor defined by equation (9) is invariant to object rotation, translation, contrast and scale change [6]. Fig.(2) shows the flowchart of calculating complex moment descriptor. 


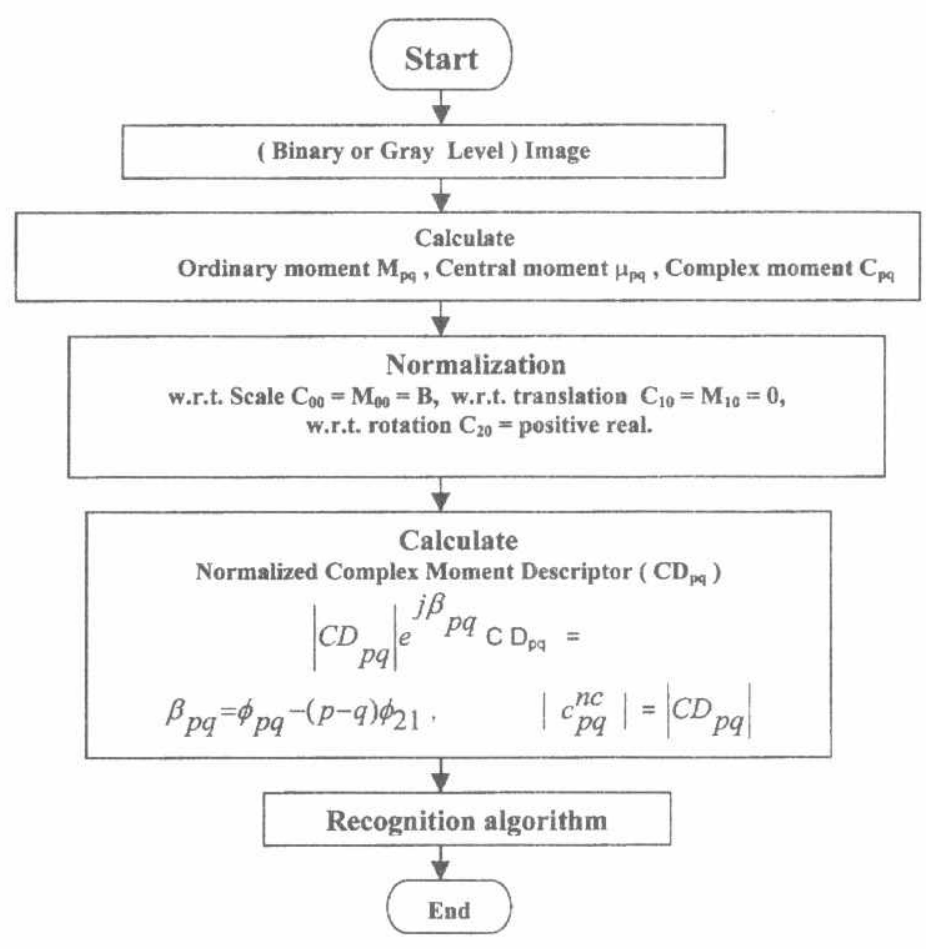

\section{Fig.(2) Flowchart of calculating complex moment descriptor}

The algorithm for calculating the CMD coefficients is simple and the output is a moment feature vector which is invariant to object transformation. The CMD approach offers computational advantages and produces better results due to its improved noise immunity in comparison to other moments.

\subsection{Complex Moment Descriptor (CMD) Classification Algorithm:}

In the generalized approach, the CMD is just a mathematical tool for forming a feature vector, which is independent of object transformation and has a good noise immunity [3]. However, the phase angle of the CMD in this case is $m$ times ( $m$ is the angular order of the reference component used) that of the corresponding complex moments at a fixed orientation in space. Despite this, classification is quite simple and the output is a complex moments feature vector, which is invariant to object transformation. The selected coefficient (for the given angular order) should have the largest value of $p+q$ such that it contains all the information present in the other coefficients with the same angular order. So instead of using all CMDs with $p>q$, up to the $5^{\text {th }}$ order only the following components of the CMD's are used for classification: $C D_{40}, C D_{31}, C D_{22}, C D_{50}, C D_{41}, C D_{32}$

A typical problem in aircraft recognition algorithm is to collect data from a physical process and classify them into known aircraft images. The known images typically 
are represented as class structures, where each class structure is described by a number of features (CMD), and stored in our knowledge base, and we are given a new data sample that has not yet been classified. We want to determine which class the sample most closely resembles.

The nearest-mean classifier calculates the distance measure by estimating the real difference angle between each of the sample and model image coefficients as follows:

$$
d=\sqrt{\sum_{p, q}\left|C D_{p q}\right|^{2}+\left|C D_{p q}^{\prime}\right|^{2}-2\left|C D_{p q}\right| *\left|C D_{p q}^{\prime}\right| \cos \left(\varepsilon_{m}\right)}
$$

where:

$C D_{p q}$ and $C D_{p q}^{\prime} \quad$ : are the amplitude of the $C M D$ of the model and sample object image respectively and, $\varepsilon_{m}=\frac{\beta_{p q}-\beta_{p q}^{\prime}}{p^{*}-q^{*}}$ in which $\left(\beta_{p q}-\beta_{p q}^{\prime}\right)$ are the phase of the CMD coefficient of order $p q$ for the model and sample respectively. Fig.(3) illustrates the flowchart of complex moment descriptor recognition algorithm.

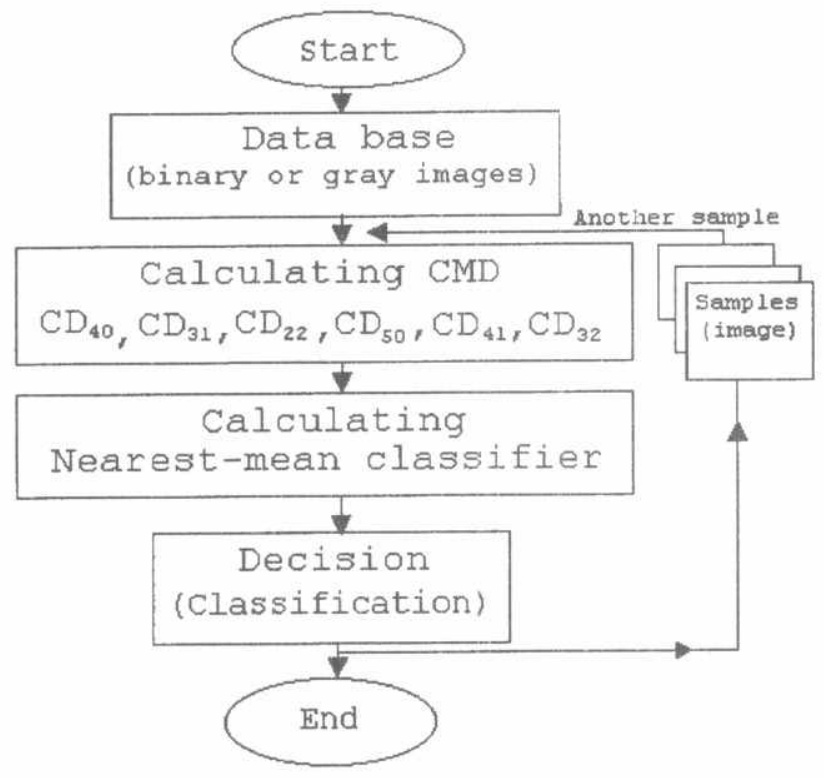

Fig. (3) Flow chart of complex moment descriptor recognition algorithm.

\section{Aircraft Models and Image Capture:}

Fig.(4) shows a four aircraft models of different types, which will be used through this study. Images of the four models are taken by camera $256 \times 256$ then scaled to be 
$64 \times 64$ [ 8 ]. Set ("I") of four groups of images are from the original aircraft images by finding the projections of each one on three levels ( horizontal, vertical, and side), as shown in Fig.(4). These projections are out database to compare the input data with it. The projections of aircraft models were used for getting of images each image is rotated by a rotation angle to represents our data ( set "II" of images ) as shown in Fig.(5).

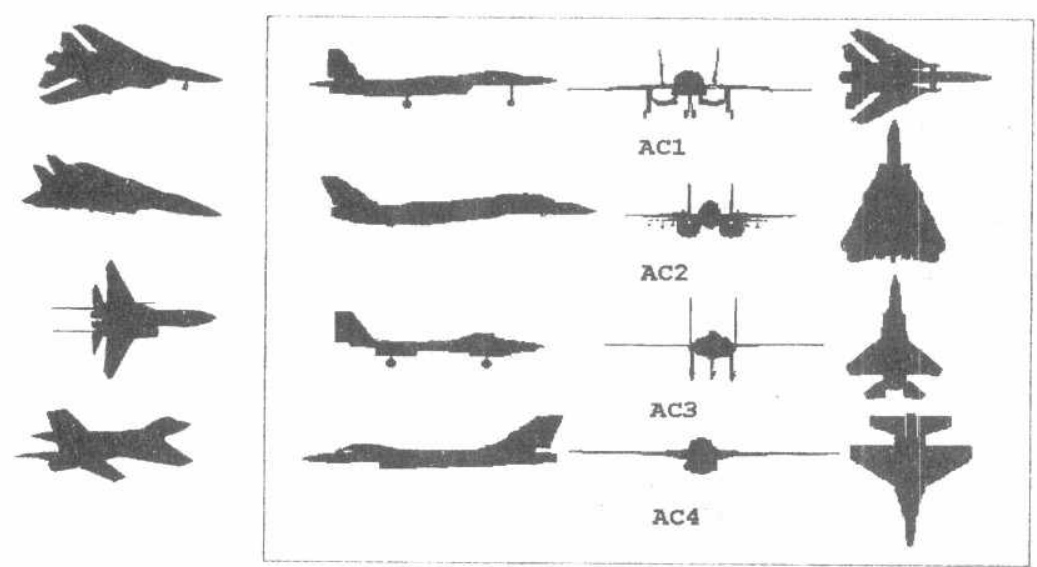

Fig.(4) A four different images of aircraft models and their projections ( horizontal, vertical, and side ).

The most common method adapted in moment based recognition algorithms is the comparison of feature vectors of a set of reference images with the given image of unknown object. The feature vectors are constituted by invariant functions of moments of different orders, so that the global shape characteristics of the image are available with the required level of detail and at the same time are invariant with respect to camera position and rotation angle [ 7,10 ]. The moment features computed from images can be used to identify the object irrespective of the position and orientation of the image in its plane. A set of moment functions of a particular image is referred to as feature vector. A set of feature vectors can be used to represent a class of patterns or a set of different views of an object.

The selection of an appropriate feature for a particular pattern matching application, is generally based on the following aspects :

1. Information content: The number and order of moments needed to adequately and unambiguously represent the shape features.

2. Robustness: Sensitivity of the components of feature vectors to image noise, spatial quantization, and intensity quantization.

3. Information redundancy: Capability of the components of feature vectors to characterize independent image features. 

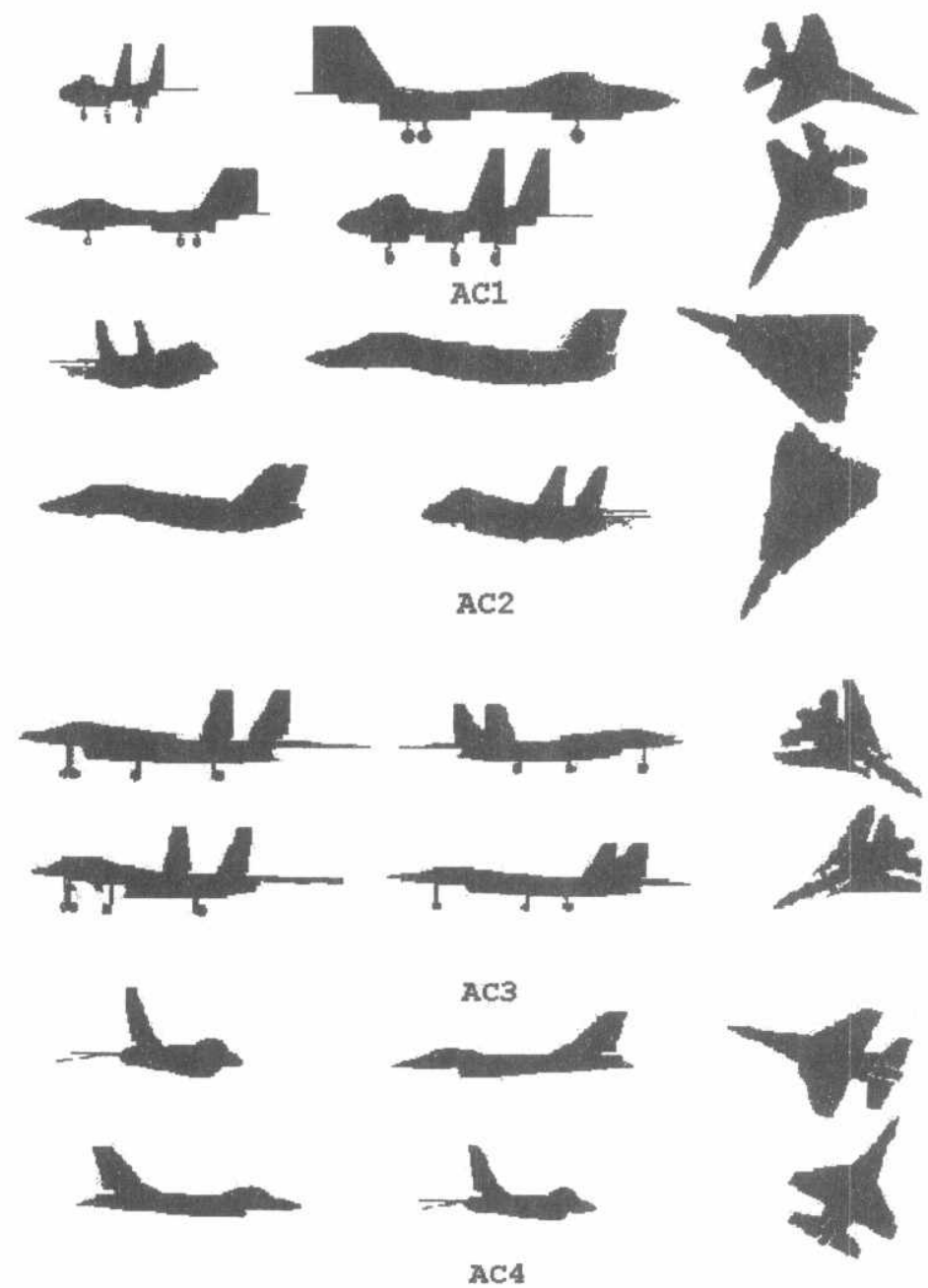

Fig.(5) Testing samples of four images with different oritations.

The feature vector computed from an image (measurement) has to be compared and matched with a class of feature vectors stored a priori (reference), to establish the correspondence between the given image (of unknown object or pattern) and a standard image. We denote a feature vector corresponding to an image $\mathrm{k}$ by: 


$$
V^{(k)}=\left[v_{1}^{(k)} v_{2}^{(k)} v_{3}^{(k)} \ldots . . v_{n}^{(k)}\right]
$$

where each component $v_{i}^{(k)}$ is typically an invariant moment function of the image. The set of all $v_{i}^{(k)}$ 's constitute the reference library of feature vectors. The images for which the reference vectors are computed and stored as above are either a set of patterns.

$$
V^{\prime}=\left[v_{1}^{\prime} v_{2}^{\prime} v_{3}^{\prime} \ldots . . v_{n}^{\prime}\right]
$$

The problem considered here is to match a feature vector of the image of an unknown pattern or object view, with the vectors in the reference library to identify the pattern or view direction. We find from [6] where the comparison study has been performed, the nearest-mean classifier method is very simple and perform the calculation in real time for that we will use it in our classification problem.

\section{Complex Neural Network $\{1,4,5]$ :}

\subsection{Complex Neural Network (CNN) Architecture:}

An CNN is a group of Artificial Neurons interconnected via an interconnection structure. The basic structure of an CNN consists of : An input layer, One or more hidden layer, Output Layer Usually many persons perform their computations in the same time (this parallel processing is analogues to the way brain work). Changing the way persons are interconnected changes the shape (Topology) of the CNN. CNNs can have many topologies. Multiple-layer feedforward network (MLFN) is the "standard" neural network model [3]. It was arguably the first practical neural network and it has maintained its lead ever since. MLFN is a general-purpose program for training and testing multiple-layer feedforward neural network. The input, hidden, and output layers may be independently defined as operating in the real or complex domain. The neural network model that we will be used in this paper has eighteen input neurons by which complex moment's features are presented to the network, and four output neurons through which the network returns its results to the world. It has eighteen hidden neurons that have no contact with the outside world. The hidden neurons are often the workhorses of the network, the means by which complicated patterns are processed in useful ways. The hidden neurons are organized into one layer Fig.(6) Shows a complex neural network model with one hidden layer. Information passes in only one direction through the network. The output of the input neurons goes to the inputs of the hidden neurons, and the outputs of the hidden neurons go to the inputs of the output neurons. Information does not pass backwards, from output toward input. There is no communication among neurons in a given layer. These are the properties that are reflected in the term feed forward The input neurons are purely hypothetical constructs. They do not really exist. They do no computations and effect no actions. They simply have outputs, and those outputs are really the inputs to the network. When we present a pattern to the network, that pattern is sent directly to the hidden neurons.

The output activation level of each neuron in the network is computed with the same two-step operation. First, its inputs from all neurons' outputs in the previous layer are individually multiplied by weights, and the sum of these weighted inputs is found. 


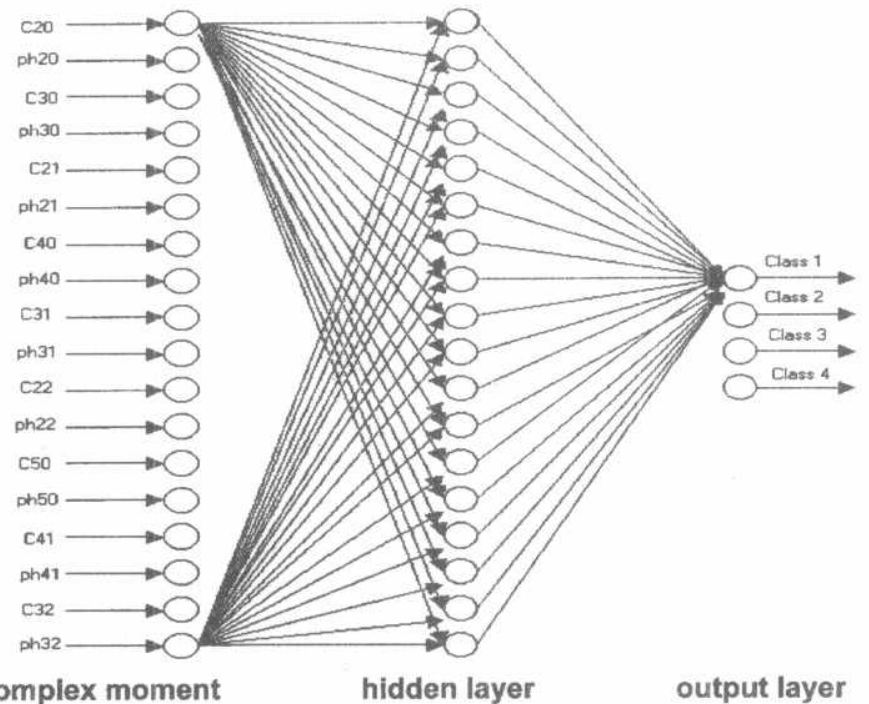

Fig. (6) A complex neural network model with one hidden layer

One additional hypothetical input, called the bias, is weighted and included in the sum. Its value is always equal to $1+0 \mathrm{i}$. The dot product operation involved in its computation is normally the major eater of time in neural network learning and execution and should always be programmed with extra care.

The second step in computing a neuron's output is that the net input is acted on by an activation function. The value of the activation function determines the output of the neuron. Traditionally, all neurons in a given layer employ the same activation function. The computation of a signal neuron's output activation given its inputs is shown pictorially in Fig.(7) where all terms in that equation are complex numbers.

$$
\text { out }=f(\text { net })=f\left(\sum_{j=0}^{n-1} a_{j} w_{j}+w_{n}\right)
$$

Where $a$ is the node input signal value

$W$ is the weight applied to each input

$f$ is the threshold value for the node 


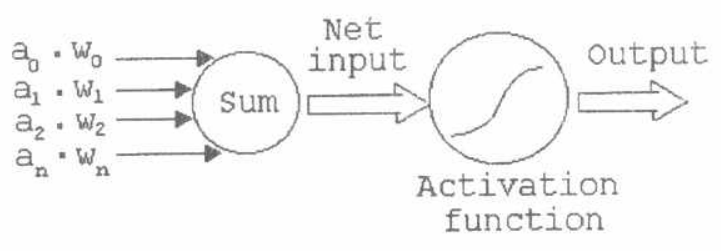

\section{Fig.(7) Computing the activation of a neuron}

There are different approaches for the implementation of complex neural network activation function. Kim [14], proposed the function:

$$
f(x+j y)=1 /\left(1+e^{-(x+j y)}\right)
$$

which is a direct substitution for the complex input. However, practical work, [14], shows that such function has violent discontinuities across its domain. This precludes its use if gradients are to be computed for learning algorithms. An alternative approach by Rosi [14] is the use of the function:

$f(x+j y)=\tanh (x)+j \tanh (y)$

In this equation squashing function is applied to the real and imaginary parts using this technique.

There is another appealing property that a function like that shown in Eq.(15). It would be nice if the activation function preserved the direction of the net input, squashing only its length. This is easily implemented. Suppose that $S(x)$ is a sigmoid function having $S(0)=0$. The hyperbolic tangent is one such function. It is clear that we will only be concerned with nonnegative value of its domain variable. Then, one can squash the length of a length of a complex number without changing its direction by means of the function shown:

$$
\begin{aligned}
& f(x+j y)=p x=j p y ; \\
& p=\frac{S\left(\sqrt{x^{2}+y^{2}}\right)}{\left(\sqrt{x^{2}+y^{2}}\right)}
\end{aligned}
$$

Master [14], reported that a choice of $S(x)=\tanh (1.5 x)$ is a good choice.

\subsection{Complex Neural Network Training Algorithm:}

Training means that, the artificial NN can modify its behavior in response to their environment, once the network is trained, the networks's response can be sensitive to minor variants in its input. Some (ANN's) are capable of abstracting the essence or the core a set of input. Extracting idealized prototypes is a highly useful ability in humans. However, (NN's) have been used into pattern recognition applications, in which conventional computerized machines do it poorly [12]. The procedure used to perform the training process is called a learning algorithm, the function of which is to modify those synaptic weights to attain a desired design objective. When an object of interest rotates the image of the object as perceived by the observer usually changes 
in a corresponding way. In order to build an object recognition system for dealing with these changes, the system must be capable of coping with this transformation of the observed signal. A primary requirement in this case is to design a classifier which output must not be affected by such transformation at its input. This classifier is called invariant classifier. There exist mainly three approaches for design of such NN classifier. In these approaches invariance can be achieved through: structure, training or feature vector of NN. Multi layer feedforword network (MLFN) is a general purpose program for training and testing multiple-layer feedforword neural network [14]. The input, hidden, and output layers may be independently defined as operating in the real or complex domain. A hybrid of simulated annealing and conjugate gradient optimization is used as the primary training algorithm. After training, validation sets may be processed for the purpose of evaluating the network's performance. The trained network may also be executed on unknown data with the results saved to a disk file. Finally the weights may be saved in a compact file format to facilitate reuse at a later date.

A hybrid of simulated annealing and conjugate gradient optimization algorithm is the standard workhouse method, it combines the global search strategy of simulated annealing with the powerful minimum-seeking conjugate (CJ) algorithm [14]. Simulated annealing is used in two separate, independent ways in this method. First it is used in a high-temperature initialization mode, centered at weights of zero, to find good starting point for $\mathrm{CJ}$ minimization. When the $\mathrm{CJ}$ algorithm subsequently converges to a local minimum, simulated annealing is called into play again. This time its goal is to escape from what may be a local minimum, so its temperature is usually lower. Also, annealing is centered about the best weights as found by the CJ algorithm. A complete flowchart for this operation is shown in Fig. (8).

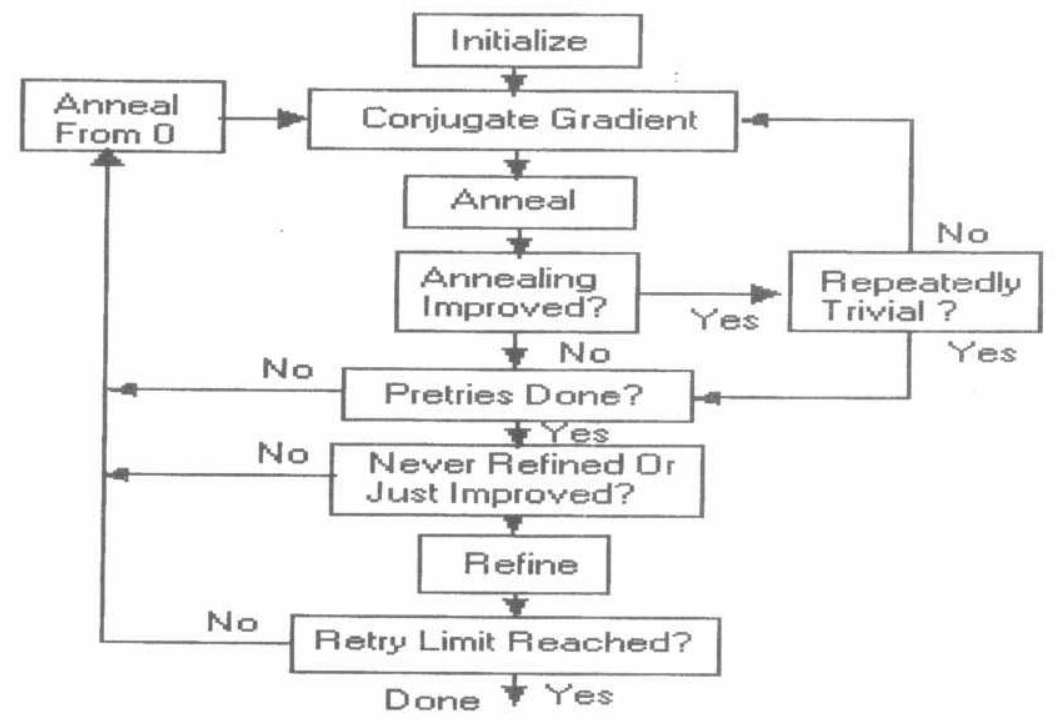

Fig.(8) . Multi layer feedforword network (MLFN) algorithm. 
In order to classify patterns using moment functions, a feature vector $V^{(k)}$ of the type given in Eq. (10) is presented at the input and the output is specified as the value of the output node $k$ being 1 and the remaining values 0 . The feature vector consists of moment invariants. The neural network is trained using $A$ hybrid of simulated annealing and conjugate gradient optimization algorithm and with different images represented by the same feature vector (i.e. images which are scaled, translated and rotated versions of the original image) specifying the same output [14].

\section{Experimental Results:}

To test the normalization factor and the CMD classification power, first, the four groups of real aircraft images (256 by 256 pixels) of Fig. (4), are taken from the image capture equipment which are then scaled to be $(65 \times 65$ pixels), these are the gray scale images. The complex moments are calculated using CMD from the binary images, these features are stored as a classes of feature vectors (a reference).

To test the classification rate of the generalized CMD classification algorithm the following steps are realized:

One hundred sample of each images from set "II", Fig.(5), after some transformation like (rotation, translation, change in scale ) were used in this experiment, where the feature vector computed from each image (measurement) has to be compared and matched with a class of feature vectors stored a priori (reference). The correspondence between the given image (of unknown object or pattern) and a standard image is established using nearest-mean classifier. The classification average rate is $91 \%$ when all samples are used as illustrated in Table 1.

One hundred - noisy sample of each projection of the four aircraft are used in this experiment, this noise is represented by probability $(p)$ for moving each of contour pixels to any of the 8-neignbor with equal chance and the classification rate is $85 \%$ when all samples are used as illustrated in Table 2. The immunity of the CMD to noise is also apparent. This immunity is due to the formation of the CMD feature vector where the amplitude of the complex moments is retained and the operation is performed on the angles. Such an approach, at least in the presence of noise, limits the deterioration of all coefficients in both phase and amplitude.

\begin{tabular}{|l|c|c|c|c|}
\hline Ip-op & Shape 1 & Shape 2 & Shape 3 & Shape 4 \\
\hline Shape 1 & 92 & 0 & 4 & 4 \\
\hline Shape 2 & 5 & 82 & 3 & 10 \\
\hline Shape 3 & 0 & 0 & 98 & 2 \\
\hline Shape 4 & 4 & 3 & 1 & 92 \\
\hline
\end{tabular}

Table 1 Matrix of CMD classification algorithm using clean images

\begin{tabular}{|l|c|c|c|c|}
\hline Ip-op & Shape 1 & Shape 2 & Shape 3 & Shape 4 \\
\hline Shape 1 & 72 & 4 & 14 & 10 \\
\hline Shape 2 & 16 & 81 & 3 & 0 \\
\hline Shape 3 & 0 & 6 & 94 & 0 \\
\hline Shape 4 & 3 & 3 & 3 & 91 \\
\hline
\end{tabular}

Table 2 Matrix of CMD classification algorithm using noisy images 
To test the performance of complex neural network (CNN) classifier based on the output of design a multiple-layer feedforword network (MLFN) for complex world problem. A complex moments are calculated from the ordinary central moments for binary images in the first phase and then the above recognition algorithm is applied for calculating normalization complex moments to be the inputs to (CNN).

A group of 240 samples for each class was considered in the learning stage. In order to make this training sample representative, it is chosen from different level of noise. Growing up technique is used to determine the size of the input and output layers. This approach shows that only 18 processing elements are necessary for the input of network. Fig. (6) show the network. The network has nearly zero error for most of the processing elements. More-over, it is clear from the figure that the network shows the most important features (those have greater shares or weights)

In test mode a different group of samples was used for each class (totally 960 samples). The CNN shows a $97 \%$ classification recognition rate and $95 \%$ in Table (3), (4) respectively

\begin{tabular}{|l|c|c|c|c|}
\hline Ip-op & Shape 1 & Shape 2 & Shape 3 & Shape 4 \\
\hline Shape 1 & 97 & 0 & 2 & 1 \\
\hline Shape 2 & 0 & 98 & 0 & 2 \\
\hline Shape 3 & 0 & 0 & 98 & 2 \\
\hline Shape 4 & 1 & 3 & 1 & 95 \\
\hline
\end{tabular}

Table 3 Matrix of CNN using clean images

\begin{tabular}{|l|c|c|c|c|}
\hline Ip-op & Shape 1 & Shape 2 & Shape 3 & Shape 4 \\
\hline Shape 1 & 97 & 1 & 1 & 1 \\
\hline Shape 2 & 7 & 90 & 3 & 0 \\
\hline Shape 3 & 0 & 6 & 94 & 0 \\
\hline Shape 4 & 3 & 1 & 0 & 96 \\
\hline
\end{tabular}

Table 4 Matrix of CNN using noisy images

\section{Conclusions \& Future work}

This paper describes a new approach to aircraft image recognition system. The method combines complex moments with the complex neural-network.

The network uses the complex moments as a feature vector that are invariant to translation, scale and rotation. This approach differs significantly from many recent aircraft image recognition system which emphasize the geometric features of objects in the scene, experimental results are given to illustrate the efficacy of this approach Complex neural networks have provided a satisfactory performance when used in the aircraft recognition system

The capability of moment invariant to represent image shapes irrespective of their rotation, translation and scale variation has been effectively used to construct feature vectors and to identify an image by feature vector correspondence. A generalization of the image normalization scheme using higher order complex moments is also described. A brief description of the complex neural network implementation 
consisting of a multi-layer preceptor with feed forward training algorithm is also given. This type of neural networks is commonly used as pattern classifiers, with complex moment invariant feature vector as input.

\section{REFERENCES:}

[1] Masrters, Timothy, "Practical Neural Network Recipes in c++" Academic Press, New York, (1993).

[2] Yen-Hao tseng etal. and Florence, "Three dimensional object representation and invariant recognition using continuous distance transform neural networks" IEEE transaction on neural network, Vol 8, pp 141-147, January (1997).

[3] A..Abo-Zaid, O.Hinton "About Moment Normalization And Complex Moment Descriptor", Proceeding of the $4^{\text {th }}$ International Conference Of Pattern Recognition, Cambridge, U.K. 1988.

[4] jianchang Mao etal.and Anil Jain, "Artificial neural networks for feature extraction and multivariate data projection" IEEE transaction on neural network, Vol. 6, pp. 296316, March (1995).

[5] A. Ravichandran and B. Yegnanarayana, "Studies on object recognition from degraded images using neural networks" IEEE transaction on neural network, Vol. 8, pp. 481-488, (1995).

[6] Ameen M. Nasory "Shape Recognition In Noisy Environment Using Fuzzy Network Approach" Ph.D., Military Technical College, Cairo, Egypt, 2000.

[7] Stavros J. Perantonis and Paulo Lisboa, "Translation, rotation, and scale invariant pattern recognition by high-order neural networks and moment classifiers," IEEE transaction on neural network, Vol. 3, pp. 242-251, March (1992).

[8] C. H. The and R. T. Chin, "On Digital Approximation of Moment Invariants," Computer Vision, Graphics, and Image Processing, Vol. 33, pp. 318-326, 1986

[9] S. A. Dudani and R. B.Mcghee ."Aircraft Identification by Moment Invariants". IEEE Transaction on Computers, Vol. C-26, No. 1, January. 1977.

[10] G.A Awcock and R. Thomas." Applied Irnage Processing". Mc Graw-Hill,Inc., 1996.

[11] Kim Schowalter "Signal And Image Processing With Neural Networks A C++ Sourcebook" Academic Press, Inc., 1994.

[12] Robert J. Schalkoff," Artificial Neural Networks", McGraw-Hill Singapore, (1997).

[13] A...Abo-Zaid,"General Purpose Feature Extraction Algorithms and Their Implementation", Phd ,Canterbury, Kent, U.K.1989.

[14] Dan W. Patterson "Artificial Newral Networks, Theory and Applications" First published 1996 by Prentice Hall. 